\title{
Comparing shared control approaches for alternative interfaces: a wheelchair simulator experiment
}

\author{
Chinemelu Ezeh ${ }^{1}$, Pete Trautman ${ }^{2}$, Catherine Holloway ${ }^{3}$ and Tom Carlson ${ }^{1}$
}

\begin{abstract}
Independent mobility is important for the selfesteem and well-being of people with mobility impairments. For people with severe disabilities, there is a body of research investigating how best to share control of motion between a person with disabilities and a "smart wheelchair". Traditionally in "shared control", the control law is a linear combination of the human's intended velocity and the path planner's velocity. However, this formulation of sharing control between a human and a machine does not guarantee safety on a theoretical level. To guarantee safety in formulating the blending of the human's input velocity and planner's velocity, we implement a practical form of probabilistic shared control formulated by Trautman. We tested this shared control by conducting experiments in a simulation where participants drive a wheelchair. The results of the experiment suggest probabilistic shared control has similar performance to linear blending in terms of significant reduction in number of collisions. However, probabilistic shared control reduced more collisions on average than linear blending did for the sip-puff switch, which is a difficult control interface to drive a wheelchair with.
\end{abstract}

\section{INTRODUCTION}

The ability to move around independently is important for self-esteem and well-being of people with mobility impairment [1]. Usually, people with mobility impairments are provided a form of assistive technology to allow them to move around such as a scooter, a manual or a powered wheelchair and scooter. People with more severe conditions such as insufficient upper body strength, are usually given a powered wheelchair as it can be used with interfaces such as a joystick that requires minimal upper body strength.

However, not everyone with severe mobility impairments can use a joystick. A clinical survey was conducted that showed commercially available interfaces are inadequate for people with severe disabilities to control a powered wheelchair [2]. In fact, novel interfaces may be needed given the variation of impairments that do not permit patients to benefit from present forms of mobility [3].

Several novel interfaces and techniques to control powered wheelchairs have been suggested in the literature. Most techniques are based on improvements in joystick control [4]-[7]. Besides joystick control, some techniques for driving a powered wheelchair employ completely new interfaces

\footnotetext{
${ }^{1}$ Chinemelu Ezeh and Tom Carlson are with Aspire Create, University College London, Royal National Orthopaedic Hospital, HA7 4LP, UK \{chinemelu.ezeh.14, t.carlson\}@ucl.ac.uk

${ }^{2}$ Pete Trautman is with Galois Inc., Portland, US peter.trautman@galois.com

3 Catherine Holloway is with the UCL Interaction Centre (UCLIC), University College London, Gower Street, WC1E 6EA, UK .hollowayeucl.ac.uk
}

(i.e. these interfaces are not yet commercially available for wheelchair control) such as the Brain-Computer Interface [8], [9]. However, there are commercially available interfaces that have been overlooked such as head-array, sip-puff switch and chin joystick. It makes sense to leverage these interfaces by combining them with algorithms and sensors to improve a wheelchair user's manoeuvring capability.

In this paper, we investigate improving the adequacy of alternative interfaces using a probabilistic approach for blending the user's trajectory with the path planner's trajectory called probabilistic shared control (PSC) and a linear blending approach. The alternative interfaces are: head-array and sippuff switch. We performed experiments in a simulation and the objective was improve driving performance using these alternative interfaces, bringing the performance as close as possible to that when using a joystick (without shared control).

Section II of the paper discusses how this paper varies from the literature. Section III introduces the path planner's implementation we adopted for our shared control. Section IV discusses our formulation of probabilistic shared control that blends velocity from our path planner with the human's intended velocity. Section V highlights our experiment to capture the performance of probabilistic shared control for the different interfaces in a simulation whilst Section VI shows the results of the experiment. Finally, Section VII discusses the results of the experiment.

\section{RELATED WORK}

The current state of the art in shared control continuous blends of the user's intended velocity and some sort of optimal velocity of a mobile robot [4], [5], [10], [11]. Generally these strategies use (variations of) the following equation to blend the user's intended velocity with the robot's velocity [12]:

$$
\begin{aligned}
& \quad \mathbf{u}^{L B}(t)=K_{h} \mathbf{u}^{h}(t)+K_{R} \mathbf{u}^{R}(t), \\
& \text { where } K_{h}+K_{R}=1,
\end{aligned}
$$

and $\mathbf{u}^{L B}$ is the linear blended velocity sent to the robot. $\mathbf{u}^{h}(t)$ and $\mathbf{u}^{R}(t)$ are the human's intended trajectory and the robot's trajectory respectively, computed at time, $t-1$ for the next time step, $t$. Different linear blending approaches compute the weight of the user's trajectory, $K_{h}$ and the weight of the path planner's command, $K_{R}$ in different ways.

However, linear blending does not guarantee safety. To account for uncertainty in user's intentions and inability to perform certain manoeuvres, probabilistic models of the user's intention have been employed [13]-[15]. Probability distributions have been used to model the actual blending of the 
human's intended velocity taken from a joystick and the path planner's velocity [16].

Here, the probability distribution of the user's intended velocity and that of the path planner are linked in a joint probability distribution that maximises the probability of obtaining a path planner's velocity that is closest to what the user intended. This planner's velocity is the resultant control law used to drive the wheelchair. Unlike linear blending, this probabilistic blending guarantees safety to the same extent that the path planner does and it accounts for uncertainty in the blending process at least on a theoretical level [12]. The final output of the shared control law used to drive the wheelchair is the planner's velocity that maximises this joint probability distribution.

In this paper, we employ a probabilistic approach to blending similar to [16]. However, we include probabilistic models for alternative interfaces: head-array and sip-puff switch.

\section{Motion Planning Using the Dynamic Window APPROACH}

The path planner of our shared control system is based on the Dynamic Window Approach (DWA) for collision avoidance. DWA works by selecting the linear and angular velocity pair $(v, \omega)$ that maximises a set of objectives whilst still ensuring that the velocity pair obey constraints imposed by the kinematic model of the wheelchair and does not lead to collisions. The objectives DWA maximises are: Heading, Clearance and Velocity. This objective can be formulated as:

$$
\begin{aligned}
(v, \omega)^{*}= & \arg \max _{(v, \omega)}(H \cdot \text { Heading }(v, \omega)+ \\
& C \cdot \text { Clearance }(v, \omega)+V \cdot \operatorname{Velocity}(v, \omega))
\end{aligned}
$$

Where $H, C, V$ are the weights indicating how much importance we ascribe to each of the objectives. The simplest of these objectives, velocity is simply the normalised translational velocity: velocity $=\frac{|v|}{v_{\max }}$

In this section, we discuss some improvements we made when implementing the other objectives. In our implementation and unlike in the original version of DWA, we needed to allow the wheelchair to drive backwards. In particular, we modified DWA so that we are able to compute the heading and clearance for reverse motion.

\section{A. Heading}

Heading is a measure of the alignment of the wheelchair's orientation, $\theta_{R}$ with a direction of the goal position. Heading for a particular velocity pair is measured at the position $\left(x_{R}, y_{R}\right)$ the robot reaches when it decelerates using its maximum deceleration one time step after moving at the velocity pair. Thrun calculated heading as $\pi$ radians minus the smaller angular distance between the orientation of the wheelchair and the bearing of the goal position $\left(x_{G}, y_{G}\right)$ from the wheelchair [17]. In our implementation, we normalise this heading and add a new heading equation for reverse motion. Mathematically, we have:

Heading $= \begin{cases}\frac{\pi-\mid\left(\text { bearing }_{R \rightarrow G}-\theta_{R_{t}}\right) \mid}{\pi}, & \text { if direction=forward } \\ \frac{-\mid\left(\text { bearing }_{R \rightarrow G}-\theta_{R_{t}}\right) \mid}{\pi}, & \text { if direction=reverse }\end{cases}$

Where bearing $\operatorname{lat}_{R}=\operatorname{atan} 2\left(y_{G}-y_{R}, x_{G}-x_{R}\right)$

The notation $\left|\theta_{1}-\theta_{2}\right|$ returns the smaller angular difference between the two angles, $\theta_{1}$ and $\theta_{2}$.

\section{B. Clearance}

Originally, clearance is measured as the minimum distance to an obstacle along a trajectory. However, this metric does not account for purely rotational trajectories [18]. To account for clearance of purely rotational trajectories, we assume the distance to the obstacle is the linear distance swept by the arc from the nearest edge of the wheelchair's body to its centre of rotation before the wheelchair collides with the obstacle. Our final clearance is then a normalised distance to obstacle. Mathematically, we have:

$$
\begin{aligned}
& \text { Clearance }(v, \omega)= \\
& \qquad \begin{cases}1, & \text { if } v=0 \wedge \omega=0 \\
\frac{d(v, \omega)}{d_{\text {max }}}, & \text { if }|v|>0 \wedge d>S \\
\frac{d=\left|\theta_{\text {col }}-\theta_{R_{t}}\right| \cdot r}{d_{\text {max }}}, & \text { if }|v|=0 \wedge|\omega|>0 \wedge d>S \\
0, & \text { if } d<S\end{cases}
\end{aligned}
$$

Where $d(v, \omega)$ is the nearest distance from an obstacle if it moves with velocity, $(v, \omega) . \mathrm{S}$ is the safety threshold we adopted as an added guard against collision and was set to $0.02 \mathrm{~m}$. $\theta_{\mathrm{col}}$. is the orientation of the wheelchair at the point of collision and $\theta_{R_{t}}$ is the current orientation of the wheelchair.

\section{Probabilistic Shared Control}

We implemented a practical form of the probabilistic shared control that was theorised in [12]. This implementation was an improvement on our first implementation detailed in [16]. In particular, we improved the autonomy performance by focussing on testing its parameters in a simulation and modifying our algorithm to perform better for driving in reverse. In our shared control, we model the interaction between the user's intention and the wheelchair's path planner as a joint probability distribution. In this case the control law or final motor commands to the wheelchair attempts to maximise this joint probability function. We formulate this joint probability function as:

$$
p\left(\mathbf{u}^{h}, \mathbf{u}^{R} \mid \overline{\mathbf{z}}_{1: t}\right)=\underbrace{\psi\left(\mathbf{u}_{t+1}^{h}, \mathbf{u}_{t+1}^{R}\right)}_{\text {coupling factor }} \underbrace{p\left(\mathbf{u}_{t+1}^{h} \mid \mathbf{z}_{1: t}^{h}\right)}_{\text {human model }} \underbrace{p\left(\mathbf{u}^{R} \mid \overline{\mathbf{z}}_{1: t}\right)}_{\text {planner's model }},
$$

where $\mathbf{u}^{h}$ is the human's intended velocity. $\mathbf{u}^{R}$ is the path planner's velocity. $\mathbf{u}^{E}$ represents the velocity of the obstacles 
in the environment. $\overline{\mathbf{z}}_{1: t}$ represents the combined measurements of the human's intentions, static obstacles and the robot's state up until time, t. $\mathbf{z}^{h}$ represents measurements of human input alone. We discuss the definition and implementation of the human model, planner's model and coupling factor below.

\section{A. Human Model}

The human model describes how we estimate the user's intended velocity from measurements of the user's input. Input interfaces could range from a joystick, an eye-gaze tracker to a brain-computer interface. In this paper, we concern ourselves with the joystick, head-array and sip-puff switch as input interfaces.

In general, we assume that the user model is given by a normal distribution around the current input measurement and did not depend on any past information [4]. The human model for the head-array and sip-puff switch is given as follows:

$$
p\left(\mathbf{u}_{t+1}^{h} \mid \mathbf{z}_{1: t}^{h}\right)_{\text {interface }}=\frac{1}{\sigma \sqrt{2 \pi}} e^{-\left(\mathbf{u}_{t+1}^{h}-\mathbf{z}_{t}^{h}\right)^{2} / 2 \sigma^{2}},
$$

where $\sigma$ is the variance of the distribution indicating the degree to which we trust that the input represents the user's true intended velocity. For the joystick, however, we assumed the user's intended velocity is equivalent to the most current user's input. Our assumption is another way of saying we completely trusted the user's input so that $\sigma \rightarrow 0$. We made this assumption for simplicity since the joystick has a high resolution of control and thus can be used to indicate intended motion with high accuracy [19]:

$$
p\left(\mathbf{u}_{t+1}^{h} \mid \mathbf{z}_{1: t}^{h}\right)_{\text {joystick }}=p\left(\mathbf{u}_{t+1}^{h} \mid \mathbf{z}_{t}^{h}\right)=\delta\left(\mathbf{u}_{t+1}^{h}-\mathbf{z}_{t}^{h}\right) .
$$

Since both the head-array and sip-puff switch can only indicate the four cardinal directions and four secondary inter-cardinal directions, we made some assumptions about the user's intended velocity to allow the user to express more velocities as intention than the eight directions permit. We assumed that when a person indicates a straight forward or backward motion, he/she strictly meant to move only in the forward or backward direction respectively. We found that assuming a range of directions around a straight motion indicated by the user turned the wheelchair much more in situations where the user clearly wanted to just go straight without turning. We, however, assumed that when the user indicated a turn, he/she would be happy with a range of directions around the indicated direction. Mathematically, we have:

$$
p\left(\mathbf{u}_{t+1}^{h} \mid \mathbf{z}_{1: t}^{h}\right)_{j}= \begin{cases}\frac{1}{\sigma_{j} \sqrt{2 \pi}} e^{-\left(\mathbf{u}_{t+1}^{h}-\mathbf{z}_{t}\right)^{2} / 2 \sigma_{j}^{2}}, & \text { if } \omega \neq 0 \\ \delta\left(\mathbf{u}_{t+1}^{h}-\mathbf{z}_{t}^{h}\right), & \text { if } \omega=0\end{cases}
$$

where $j \in\{$ head-array, sip-puff switch $\}$

Since the sip-puff switch is a two-switch interface and thus more difficult to use than the head-array, which is a threeswitch interface, we let $\sigma_{\text {sip-puff switch }}>\sigma_{\text {head-array }}$ [19].

\section{B. Planner's Model}

The planner's model is derived from our modified version of the dynamic window approach discussed in section III. The Dynamic window approach produces a set of trajectories that are safe and can be reached by the robot within next time step. These trajectories are weighted based on their heading to the goal location, distance from obstacle also known as clearance and velocity to form the probability distribution of the autonomy, $p\left(\mathbf{u}^{R} \mid \overline{\mathbf{z}}_{1: t}\right)$. Mathematically, the probability of trajectory, $i$ is given as:

$$
\begin{gathered}
p\left(\mathbf{u}^{R_{i}} \mid \overline{\mathbf{z}}_{1: t}\right)=H \cdot \text { Heading }\left(\mathbf{u}^{R_{i}}\right)+C \cdot \operatorname{Clearance}\left(\mathbf{u}^{R_{i}}\right)+ \\
V \cdot \operatorname{Velocity}\left(\mathbf{u}^{R_{i}}\right)
\end{gathered}
$$

Where $H, C, V$ are the constant weightings for heading, clearance and velocity respectively and were tuned empirically to favour sharp turns around obstacles. We normalised the probability function by ensuring that $H, C, V$, heading, clearance and velocity, each are scaled so that they are in the range of 0 to 1 .

Another modification we made to our already modified planner was to specify the goal pose. The goal pose was simply $1 \mathrm{~m}$ towards the direction indicated by the user's commands. This implementation of a goal was sufficient for our shared control when driving the wheelchair whilst avoiding obstacles.

\section{Coupling Factor}

The coupling factor, $\psi\left(\mathbf{u}^{h}, \mathbf{u}^{R}\right)$ represented the degree to which the human's intended velocity and the path planner's velocity agree or are similar. Mathematically this is:

$\psi\left(\mathbf{u}_{t+1}^{h}, \mathbf{u}_{t+1}^{R}\right)=\exp \left(-\frac{1}{2 \gamma}\left(\mathbf{u}_{t+1}^{h}-\mathbf{u}_{t+1}^{R}\right)\left(\mathbf{u}_{t+1}^{h}-\mathbf{u}_{t+1}^{R}\right)^{\top}\right)$.

Parameter $\gamma$ control how strongly the human's input $\mathbf{u}^{h}$ and the autonomy $\mathbf{u}^{R}$ agree and was set empirically. A large gamma value permits more disagreement between the human's intended velocity and the path planner's velocity than a smaller value.

\section{Resultant Control Law}

The control law or velocity used to drive the wheelchair, $\mathbf{u}_{P S C}$ from our probabilistic shared control is the path planner's velocity that maximises the joint probability distribution highlighted in equation IV.1. Mathematically, we have:

$$
\begin{aligned}
& \mathbf{u}_{P S C}(t+1)=\mathbf{u}_{t+1}^{R *} \text {, where } \\
& \left(\mathbf{u}_{t+1}^{h *}, \mathbf{u}_{t+1}^{R *}\right)= \\
& \quad \underset{\left(\mathbf{u}_{t+1}^{h}, \mathbf{u}_{t+1}^{R}\right)}{\arg \max } \psi\left(\mathbf{u}_{t+1}^{h}, \mathbf{u}_{t+1}^{R}\right) p\left(\mathbf{u}_{t+1}^{h} \mid \mathbf{z}_{1: t}^{h}\right) p\left(\mathbf{u}^{R} \mid \overline{\mathbf{z}}_{1: t}\right)
\end{aligned}
$$

\section{Simulation EXPERIMENT}

In our experiment, we compared performance of users manoeuvring a wheelchair (robot) using our probabilistic shared control to the performance of a standard linear blending found in the literature and to performance when using no shared control [20]. We made the comparison for the joystick, headarray and sip-puff switch. 


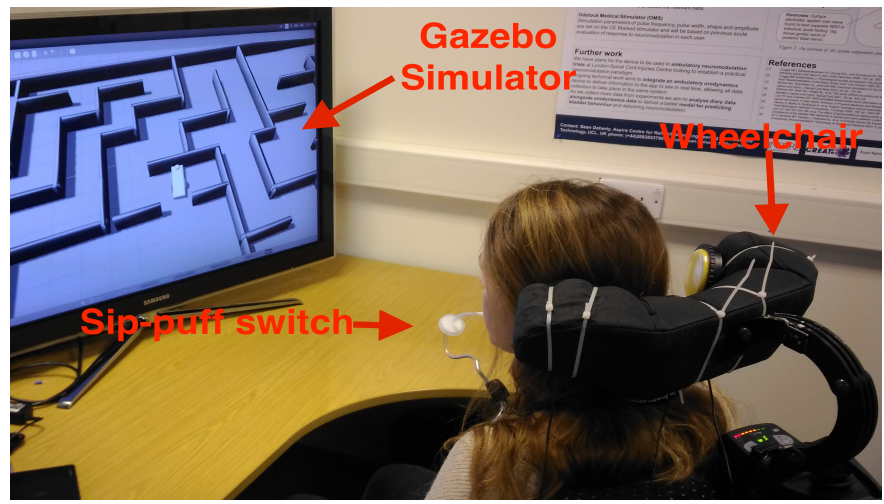

Fig. 1: Image depicting the experiment setup. The participant has a sip-puff switch in her mouth and is sat on a stationary wheelchair whilst controlling a wheelchair (robot) in the simulation.

\section{A. State of the Art for Linear Blending}

The linear blending used in this experiment is based on a similar approach to that found in the literature [4]. Here, the weights of the user's intended velocity and the planner's velocity were modulated based on how close the wheelchair was to obstacles. The closer the wheelchair got, the more control was given to the planner. Mathematically, we have:

$$
\begin{aligned}
\mathbf{u}_{t+1}^{R} & =\underset{u}{\arg \max } p\left(\mathbf{u}_{t+1}^{R} \mid \overline{\mathbf{z}}_{1: t}\right) \\
\mathbf{u}_{t+1}^{h} & =\underset{u}{\arg \max } p\left(\mathbf{u}_{t+1}^{h} \mid \mathbf{z}_{1: t}^{h}\right) \\
\alpha & =e^{-C_{L B} \operatorname{Clearance}\left(\mathbf{u}_{t+1}^{R}\right)} \\
\mathbf{u}_{t+1}^{L B} & =(1-\alpha) \mathbf{u}_{t+1}^{h}+\alpha \mathbf{u}_{t+1}^{R}
\end{aligned}
$$

Clearance is the same as defined in Section III-B. C is a constant tuned empirically. We set it to give the planner a very high degree of control near obstacles.

\section{B. Protocol}

All experiments have been examined and approved by the Research Ethics Committee of University College London (ref. 6545/003) and have been performed in accordance with the ethical standards laid out in the 1964 Declaration of Helsinki.

The participants were asked to drive a virtual wheelchair (robot) in the virtual world using three interfaces, a joystick, head-array and a sip-puff switch all attached to our physical wheelchair. The participants sat on our physical wheelchair, which was stationary for the duration of the experiment.

The experiment consisted of nine conditions. For each of the three interfaces, participants drove the wheelchair with three types of assistance: Our probabilistic shared control, a standard linear blending control and no assistance. The order of the conditions were pseudo-randomised for each participant. Furthermore, for each condition, the participant was given up to 10 mins to become familiar with using the interface and the assistance. Each participant ran a single trial for each condition, which lasted for about 5 mins.

We recruited 4 and 9 able-bodied men and women all between ages 23-30 years as participants who attended with no monetary incentives given to them. However, only 3 men and 7 women were able to complete the task with the sip-puff switch and so we used only the data from the ten participants who were able to complete all the experiments to have a balanced data set.

\section{Assessment Course}

The assessment course (see Figure 1) was designed in a Gazebo simulation, which interfaces nicely with the Robotics Operating System (ROS) that our shared control was implemented upon [21], [22]. The task on this assessment course essentially consisted of manoeuvring the wheelchair around a corridor, which was tight in several places, then going into a free space before finally going through a narrow door way [23]. We ensured that the assessment course had a balanced number of turns so as to be unbiased.

\section{Performance Metrics}

The performance metrics we employed to assess how well our shared control worked were split into objective metrics and subjective metrics. We briefly discuss how we use these metrics.

a) Objective Metrics: Ideally, driving with a better performing control will take shorter distance, less time for a task and will result in less collisions. Although in reality, different users may prefer to move different paces and make take different paths to complete a task.

b) Subjective Metrics: Self-assessed metrics of performance can be used to evaluate shared control from the perspective of the participant [24]. Perceived workload is captured by the NASA Task Load Index[25], [26]. Better performing shared control should reduce the perceived workload in comparison to other shared control. The USE Questionnaire captures how well a user is satisfied with the shared control as we want users to be at least somewhat content using our shared control [27].

\section{RESUlts}

We used ANOVA to analyse all the results of our experiment. Our alpha level was 5\%. Overall, PSC and linear blending had similar performance as they both significantly reduced the number of collisions when driving through a corridor without compromising on any other performance metric. In this section, we show in more detail the objective and subjective results.

\section{A. Objective Results}

For the sip-puff switch, PSC and linear blending both significantly reduced only the number of collisions (Figure 3) without any significant change to distance travelled and task duration compared to no assistance. Number of collisions when using sip-puff switch were $29.00 \pm 22.50$ for no assistance, $5.36 \pm 3.47$ for PSC and $9.4 \pm 14.06$ for linear blending. Collisions in PSC were caused by imperfect modelling of the wheelchair control near obstacles as our planner worked with occupancy grids and the grid size was comparable to minimum 


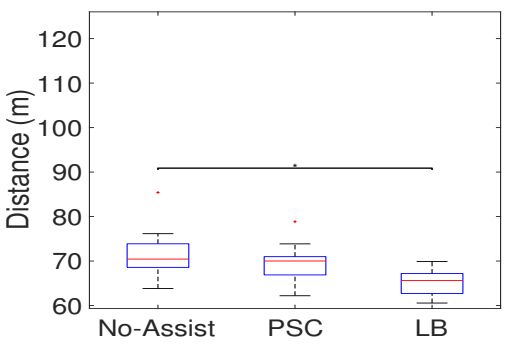

(a) PSC did reduced distance but linear blending significantly reduced distance. $(* \mathrm{p}<0.05)$.

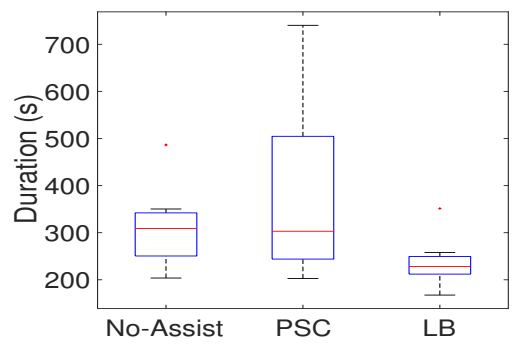

(b) PSC did not significantly increase task duration

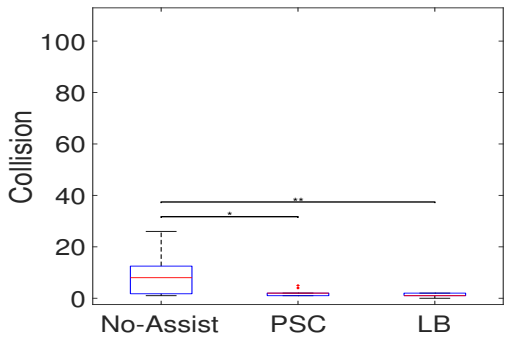

(c) Both PSC and linear blending significantly reduced number of collisions $(* \mathrm{p}<0.05$, $* * \mathrm{p}<0.01)$

Fig. 2: Participants controlling a wheelchair in a simulation using a head-array with no assistance (no-assist), probabilistic shared control (PSC) and linear blending (LB).

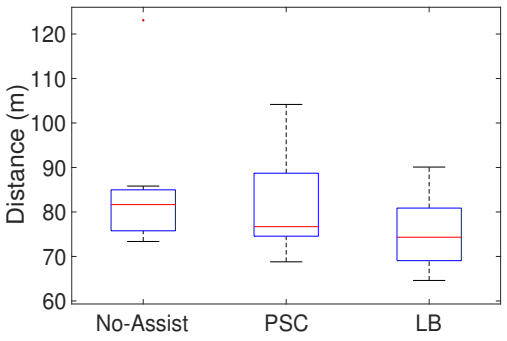

(a) No significant different amongst all types of assistance (no assistance, PSC and linear blending) for distance travelled.

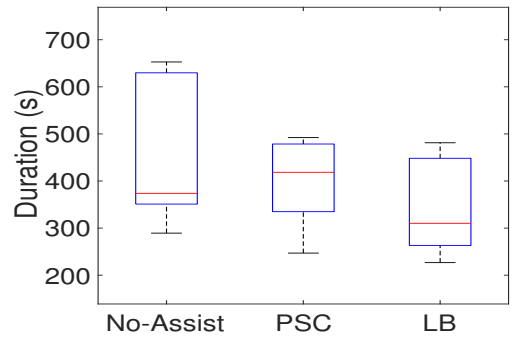

(b) Task duration did not differ significantly amongst all types of assistance.

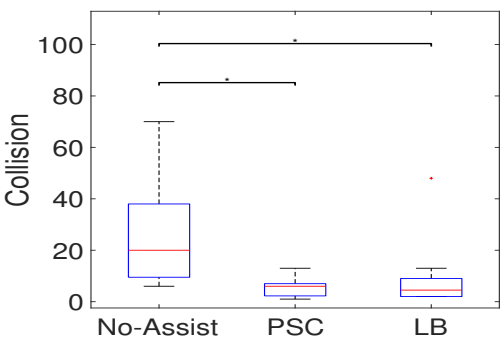

(c) Both PSC and linear blending significantly reduced the number of collision for the sip-puff switch $(* \mathrm{p}<0.05)$

Fig. 3: Participants controlling a wheelchair in a simulation using a sip-puff switch with no assistance (no-assist), probabilistic shared control (PSC) and linear blending (LB).

clearance needed to manoeuvre through tight spaces along the corridor of the simulation.

For the head-array (see Figure 2), PSC (2.20 \pm 1.32$)$ significantly reduced the number of collisions compared to no assistance $(8.55 \pm 8.09)$. Linear blending significantly reduced both the number of collisions $(1.33 \pm 0.71)$ and the distance travelled $(65.25 \mathrm{~m} \pm)$ for the head-array compared with no assistance. Distance travelled for no assistance using headarray was $71.85 \pm 6.18 \mathrm{~m}$.

For the joystick, shared control did not significantly improve driving performance. We believe this non-improvement is because our participants being able-bodied, found the joystick adequate for control in a simulation.

Compared with using the joystick with no shared control, using the head-array with all types of assistance did not significantly change clearance and number of collisions. However, for the sip-puff switch only probabilistic shared control improves the clearance and reduces number of collisions compared with using a joystick with no shared control. Probabilistic shared control, thus, brings performance in terms clearance and number of collision when using the sip-puff switch to the level achieved when using of a joystick without shared control.

Finally, no type of shared control reduced the distance travelled and task completion time of both the head-array and sip-puff switch in comparison to the joystick used with no shared control.

\section{B. Subjective Results}

For the sip-puff switch, participants generally were more satisfied with PSC compared to no assistance. In particular from the USE questionnaire, participants rated PSC as significantly more useful than no assistance. They rated a preference for linear blending between that of PSC and noassistance so that the preference of linear blending was not significantly different from the preference of the other two types of assistance.

For the head-array, participants generally preferred linear blending to both PSC and no assistance. Participants rated linear blending as significantly more favourably for statements such as "I quickly became skilful with it" and "I am satisfied with it".

For the joystick, the USE Questionnaire did not indicate any significant difference between the types of assistance.

Work load measured by the NASA-TLX also did not indicate any significant difference when employing the different types of assistance for any of the interfaces. This suggests that perceived work load did not vary with the type of assistance used.

\section{DISCUSSION}

PSC reduced collisions the most for the sip-puff switch, which was the most difficult interface to user [19]. Linear blending however, reduced the distance travelled and task duration the most for all interfaces. In addition, linear blending 
reduced collisions the most for the joystick and head-array but only slightly more than PSC. This observation, coupled with the results of the subjective evaluation of satisfaction, may indicate that participants found linear blending easier to learn to use as it did not restrict motion as much as PSC did.

A major difference of PSC from linear blending is that in PSC, the planner's velocity is the resultant control law. As a result, with PSC, we can guarantee safety to the extent that our path planner generates safe commands and this safety is entirely dependent on the planner's implementation and not on the user's input.

More work is needed, however, to bring PSC's performance to the same level as Linear blending's performance.

\section{CONCLUSION}

In this paper, we have compared a practical form of probabilistic shared control to a state-of-the-art shared control based on linear blending when driving using alternative interfaces: head-array and sip-puff switch. In probabilistic shared control, the probability distribution of the user's intended velocity and that of the path planner are linked in a joint probability distribution that maximises the probability of obtaining a path planner's velocity that is closest to what the user intended. In our experiments conducted, probabilistic shared control reduced more collisions on average compared to linear blending without compromising on distance travelled and task duration time. This research is a promising step towards a validating a mathematically principled structure, that accounts for uncertainty in the blending stage of shared control.

\section{ACKNOWLEDGMENT}

The authors would like to thank the UCL Grand Challenge Scholarship for funding this research and would also like to thank all the participants for taking part in the experiment.

\section{REFERENCES}

[1] A Davies, LH De Souza, and AO Frank. Changes in the quality of life in severely disabled people following provision of powered indoor/outdoor chairs. Disability \& Rehabilitation, 25(6):286-290, 2003.

[2] Linda Fehr, W Edwin Langbein, and Steven B Skaar. Adequacy of power wheelchair control i terfaces for persons with severe disabilities: A clinical survey. Journal of rehabilitation research and development, 37(3):353-360, 2000.

[3] Richard C Simpson, Edmund F LoPresti, and Rory A Cooper. How many people would benefit from a smart wheelchair? Journal of rehabilitation research and development, 45(1):53, 2008

[4] Tom Carlson and Yiannis Demiris. Collaborative control for a robotic wheelchair: evaluation of performance, attention, and workload. IEEE Transactions on Systems, Man, and Cybernetics, Part B (Cybernetics), 42(3):876-888, 2012.

[5] Alexander Hüntemann, Eric Demeester, Gerolf Vanacker, Dirk Vanhooydonck, Johan Philips, Hendrik Van Brussel, and Marnix Nuttin. Bayesian plan recognition and shared control under uncertainty: Assisting wheelchair drivers by tracking fine motion paths. In Intelligent Robots and Systems, 2007. IROS 2007. IEEE/RSJ International Conference on, pages 3360-3366. IEEE, 2007.

[6] Emmanuel Vander Poorten, Eric Demeester, Eli Reekmans, Johan Philips, Alexander Hüntemann, and Joris De Schutter. Haptic obstacle avoidance for intuitive powered wheelchair navigation.

[7] Dirk Vanhooydonck, Eric Demeester, Alexander Hüntemann, Johan Philips, Gerolf Vanacker, Hendrik Van Brussel, and Marnix Nuttin. Adaptable navigational assistance for intelligent wheelchairs by means of an implicit personalized user model. Robotics and Autonomous Systems, 58(8):963-977, 2010.
[8] Tom Carlson and Jose del R Millan. Brain-controlled wheelchairs: a robotic architecture. IEEE Robotics and Automation Magazine, 20:6573, 2013.

[9] Ferran Galán, Marnix Nuttin, Eileen Lew, Pierre W Ferrez, Gerolf Vanacker, Johan Philips, and J del R Millán. A brain-actuated wheelchair: asynchronous and non-invasive brain-computer interfaces for continuous control of robots. Clinical Neurophysiology, 119(9):2159-2169, 2008.

[10] Anca D Dragan and Siddhartha S Srinivasa. A policy-blending formalism for shared control. The International Journal of Robotics Research, 32(7):790-805, 2013

[11] L. Devigne, V. K. Narayanan, F. Pasteau, and M. Babel. Low complex sensor-based shared control for power wheelchair navigation. In IEEE/RSJ International Conference on Intelligent Robots and Systems (IROS), pages 5434-5439, Oct 2016.

[12] Pete Trautman. Assistive planning in complex, dynamic environments: a probabilistic approach. In Systems, Man, and Cybernetics (SMC), 2015 IEEE International Conference on, pages 3072-3078. IEEE, 2015.

[13] A. Huntemann, E. Demeester, E. V. Poorten, H. Van Brussel, and J. De Schutter. Probabilistic approach to recognize local navigation plans by fusing past driving information with a personalized user model. In 2013 IEEE International Conference on Robotics and Automation, pages 4376-4383, May 2013.

[14] E. Demeester, A. Huntemann, J. del R. Millan, and H. Van Brussel. Bayesian plan recognition for brain-computer interfaces. In 2009 IEEE International Conference on Robotics and Automation, pages 653-658, May 2009.

[15] R. C. Simpson and S. P. Levine. Automatic adaptation in the navchair assistive wheelchair navigation system. IEEE Transactions on Rehabilitation Engineering, 7(4):452-463, 1999.

[16] Chinemelu Ezeh, Pete Trautman, Louise Devinge, Valentin Bureau, Marie Babel, and Tom Carlson. Probabilistic vs linear blending shared control for wheelchair driving. In IEEE International Conference on Rehabilitation Robotics, 2017. IEEE, in review.

[17] D. Fox, W. Burgard, and S. Thrun. The dynamic window approach to collision avoidance. IEEE Robotics Automation Magazine, 4(1):23-33, Mar 1997.

[18] Pablo Inigo-Blasco, Fernando Diaz-del Rio, Saturnino Vicente Diaz, and Daniel Cagigas Muniz. The shared control dynamic window approach for non-holonomic semi-autonomous robots. In ISR/Robotik 2014; 41st International Symposium on Robotics; Proceedings of, pages 1-6. VDE, 2014.

[19] Chinemelu Ezeh, Catherine Holloway, and Tom Carlson. MoRe-T2 (mobility research trajectory tracker): validation and application. Journal of Rehabilitation and Assistive Technologies Engineering, 3:1-13, 2016.

[20] Tom Carlson and Yiannis Demiris. Human-wheelchair collaboration through prediction of intention and adaptive assistance. In Robotics and Automation, 2008. ICRA 2008. IEEE International Conference on, pages 3926-3931. IEEE, 2008.

[21] N. Koenig and A. Howard. Design and use paradigms for gazebo, an open-source multi-robot simulator. In 2004 IEEE/RSJ International Conference on Intelligent Robots and Systems (IROS) (IEEE Cat. No.04CH37566), volume 3, pages 2149-2154 vol.3, Sept 2004.

[22] Morgan Quigley, Ken Conley, Brian P. Gerkey, Josh Faust, Tully Foote, Jeremy Leibs, Rob Wheeler, and Andrew Y. Ng. Ros: an open-source robot operating system. In ICRA Workshop on Open Source Software, 2009.

[23] R Lee Kirby, Janneke Swuste, Debbie J Dupuis, Donald A MacLeod, and Randi Monroe. The wheelchair skills test: a pilot study of a new outcome measure. Archives of Physical Medicine and Rehabilitation, 83(1):10-18, 2002.

[24] T. Carlson and Y. Demiris. Collaborative control for a robotic wheelchair: Evaluation of performance, attention, and workload. IEEE Transactions on Systems, Man, and Cybernetics, Part B (Cybernetics), 42(3):876-888, June 2012.

[25] Sandra G Hart and Lowell E Staveland. Development of NASATLX (Task Load Index): Results of empirical and theoretical research. Advances in psychology, 52:139-183, 1988.

[26] Håkan Alm and Lena Nilsson. The effects of a mobile telephone task on driver behaviour in a car following situation. Accident Analysis \& Prevention, 27(5):707-715, 1995.

[27] Arnold M Lund. Measuring usability with the use questionnaire12.". Usability interface, 8(2):3-6, 2001. 